\title{
A Unified Multimedia Database System to Support Telemedicine
}

\author{
Chung-Chih Lin, Student Member, IEEE, Jeng-Ren Duann, Student Member, IEEE, Chien-Tsai Liu, \\ Heng-Shuen Chen, Jenn-Lung Su, Member, IEEE, and Jyh-Horng Chen, Member, IEEE
}

\begin{abstract}
A unified approach to managing multimedia medical databases in a telemedicine system is proposed. In order to manage, search, and display patient information more efficiently, we define a patient information package (PIP) as a concise data set of a patient's medical information from each visit. By means of PIP's, both patient-oriented and problem-oriented query strategies, which are most frequently used in daily clinical practice and medical education, can be accommodated. We also provide a unified methodology for accessing various types of patient medical records as well as design two types of user interfaces, high-quality data display and web-based interface, for different medical service purposes. The PIP-based management of databases has been successfully implemented between the National Taiwan University (NTUH), Taipei, and the Chinshan health care center, Chinshan, Taiwan, for teleconsultation, telediagnosis, and tele-education.
\end{abstract}

Index Terms - Medical database, telemedicine, World Wide Web (WWW).

\section{INTRODUCTION}

$\mathbf{D}^{-10}$ UE to shortages of medical resources and lack of opportunities for continuing education, physicians may be reluctant to serve in rural areas or geographically isolated regions. Therefore, people who live in these areas may receive lower quality medical care than those who live in urban areas. It is very important to develop a telemedicine system for improving the quality of medical services and providing more educational opportunities to physicians in rural areas [1]-[4].

Telemedicine can be defined as the providing of medical services over a distance. When telemedicine service requires patient history, medical images, and related information, telemedicine and the Picture Archiving and Communication System (PACS) become very similar [5]. Based on PACS [6]-[11], an integrated telemedicine system must consist of the following five subsystems:

Manuscript received March 13, 1998; revised July 6, 1998. This work was supported by the Grant "An NII pilot study of tele-medicine" from the Department of Health, Executive Yuan, Taiwan, R.O.C.

C.-C. Lin and J.-H. Chen are with the Institute of Electrical Engineering, National Taiwan University, Taipei, 106 Taiwan, R.O.C. (e-mail: chen@me.ee.ntu.edu.tw).

J.-R. Duann is with the Institute of Applied Physics, Chung-Yuan University, Chungli, Taiwan, R.O.C..

C.-T. Liu is with the Department of Information Systems, National Taiwan University Hospital, Taipei, 106 Taiwan, R.O.C.

H.-S. Chen is with the Department of Medical Informatics College of Medicine, National Taiwan University, Taipei, 106 Taiwan, R.O.C..

J.-L. Su is Institute of Biomedical Engineering, Chung-Yuan University, Chungli, Taiwan, R.O.C.

Publisher Item Identifier S 1089-7771(98)08041-8.
1) acquisition subsystem;

2) viewing subsystem;

3) teleconferencing subsystem;

4) communication subsystem;

5) database management subsystem.

The acquisition subsystem collects multimedia information [12] and converts it to a standard format (e.g., DICOM 3.0 [13]). The viewing subsystem displays and manipulates the images and other patient medical information [14]-[15]. The teleconferencing subsystem, which is not usually included in a PACS, allows face-to-face interactive dialog between physicians in rural areas and medical centers [16]-[18]. The communication subsystem includes local area networks (LAN's) and a wide area network (WAN) to transmit and receive data [19]-[21].

Patient medical information consists of chief complaint, history of illness, results of physical examination, laboratory tests, and diagnostic images. This information may be of the following types: text, voice, still image [e.g., x-ray, computed tomography (CT), or magnetic resonance imaging (MRI)], and dynamic video (e.g., videoesophagogram, cardioangiography, sonography, and endoscopy) [22]-[24]. Thus, it is essential to design a medical information database for managing a huge amount of heterogeneous data. In some studies [14], [26]-[28], textual data were managed by a relational database management system and image data were managed by the operating system itself using external files. However, this approach may complicate archiving operations and introduce an inconsistency problem while concurrently accessing the image data [29]. The dynamic video may be stored and managed using analog videotapes [30], [31] that are usually archived sequentially in a specific location. This management approach may make it difficult to access the videotapes and share them simultaneously. Moreover, it may perplex the integration of video with text and images in a telemedicine system.

To solve these problems, we propose a data management methodology by which medical information can be organized as a patient information package (PIP) based on the patient's complaint as well as his/her medical history. We also support a unified interface for manipulating and accessing the different types of medical information mentioned above. The PIP-based management of medical databases and the user interface have been implemented as major components of a telemedicine system connecting National Taiwan University Hospital (NTUH), Taipei, and the Chinshan healthcare center, Chinshan, Taiwan.

This paper is organized as follows. In Section II, we describe telemedicine system services, conceptual database 
models, database implementation, and user interface design. In Section III, two different applications are presented. Section IV provides some concluding remarks and areas where further work is needed.

\section{System ANALYSIS AND DESIGN}

\section{A. Telemedicine System Services}

In this paper, we have developed a telemedicine system that supports teleconsultation, telediagnosis, and tele-education. In teleconsultation, medical specialists at a medical center provide second opinions to rural physicians who have referred their patients to the specialists. The rural physicians and the specialists share the patient's medical records and discuss the symptoms of the patient's conditions interactively. The patient's final diagnosis is reached following discussion between the physicians. In teleconsultation, a synchronous two-way videoconferencing system and a document-sharing mechanism are needed to allow rural physicians to send their patient's medical information to specialists and engage in face-to-face conversation.

In telediagnosis, similar to teleconsultation, the specialist makes a diagnosis based on the received information. The major difference between telediagnosis and teleconsultation is that the former requires high-quality data and images to achieve an accurate diagnosis, while the latter requires a synchronously interactive conference environment. Telediagnosis can be performed asynchronously. That is, the specialist can make the diagnosis at his/her convenience and then forward the diagnosis report to the rural physician.

In tele-education, a rural physician playing a student role obtains advanced medical expertise from the specialists. There are two ways to deliver education to rural physicians. First, knowledge may be delivered from the specialist in a faceto-face manner through teleconferencing. For this reason, a real-time videoconferencing system is required for interactive communication. Second, the medical teaching materials may be organized and converted to a digital multimedia textbook presented on the World Wide Web (WWW). A network discussion panel may also be created for exchanging ideas and discussing problems among students and teachers. Rural physicians can access these materials and educate themselves via the Internet. In this operational mode, an authoring tool for compiling teaching materials and a userfriendly interface for browsing and discussing the multimedia textbook are required.

In order to meet the requirements of teleconsultation, telediagnosis, and tele-education simultaneously, patient medical records and the associated images must be organized in such a way that a physician can access the database based on a patient's clinical history and a medical student can access the database based on particular cases (clinical problems). It means the database must meet different purposes by providing both patient-oriented data folders and problem-oriented data folders. A patient-oriented data folder is used to store the medical records of a single patient; a problem-oriented data folder is used to store the medical records of one specific case.

\section{B. Conceptual Databases Models}

In clinical practice, a physician makes a diagnosis and treatment plan not only based on the patient's current situation, but also on a review of the patient's history and references in similar disease symptoms. Traditional medical databases are constructed according to the type of material in the records. Records, laboratory data, physicians' notes, consultant comments, and diagnostic medical images from different sources were managed in separate files. Although this management method is relatively easy to maintain, it is difficult to trace the history of particular problem. To resolve this difficulty, we have defined a PIP as a concise data set containing all of the medical diagnostic information.

After a patient visit, the physician will create a PIP according to the clinical problem. An example of PIP-based medical records is shown in Table I. A PIP data set is described by the tuple of $\operatorname{PIP}(I, N, P, S, L)$, where $I$ is the series number (PIPID). It is an increasing number for each patient's PIP's. $N$ is the patient's chart number (ChartNo), and $P$ is the unique number of the clinical problem (ProblemID). In order to trace the progress of therapies, the PIP has $S$ property to describe the processing state and $L$ property to link the previous PIP. $S$ property has four different states to describe the progress of therapy, including BEGINNING $(B)$ state, FOLLOW-UP $(F)$ state, ENDING $(E)$ state, and CHANGING $(C)$ state. $L$ is the $I$ value of the previous related PIP (PreviousPIPID). When a new problem happens in a visit, the physician will create a $B$ state PIP. Its $L$ value is set to NULL and can be signified by the tuple of $\operatorname{PIP}\left(I_{x}, N_{y}, P_{z}, B\right.$, NULL). For example, the $S$ property of this $\operatorname{PIP}(1,1,1, B$, NULL) in Table I is $B$ state. If an old problem is not yet solved, the physician generates an $F$ state PIP. The $L$ value of $F$ state PIP is the $I$ value of the last PIP with the same clinical problem. Its general form is expressed by the tuple of $\operatorname{PIP}\left(I_{x}, N_{y}, P_{z}, F, L_{m}\right)$, where $L_{m}=\left\{\max (I) \mid N=N_{\text {same ChatNo }}\right.$ and $P=P_{\text {same problem }}$ for all $\operatorname{PIP}(I, N, P, S, L)\}$. When an old clinical problem is solved in this visit, an $E$ state $\operatorname{PIP}, \operatorname{PIP}\left(I_{x}, N_{y}, P_{z}, E, L_{m}\right)$, is generated. The setting mechanism of $L$ value is similar to $F$ state PIP. In Table I, $\operatorname{PIP}(5,1,3, F, 2)$ and $\operatorname{PIP}(3,1,1, E, 1)$ are $F$ state PIP and $E$ state PIP, respectively. If a complication problem of an old problem or a new diagnostic code is changed due to new assessment, a $C$ state PIP is created. Although both $C$ state PIP and $B$ state PIP are applied to designate a new clinical problem, the $L$ value of $C$ state PIP is not a NULL. Its value is the $I$ value of the related problem PIP in this visit. It is represented by the tuple of $\operatorname{PIP}\left(I_{x}, N_{y}, P_{z}, C, L_{m}\right)$, where $L_{m}=\left\{I \mid N=N_{\text {same CharNo }}\right.$ and $P=P_{\text {related problem }}$ in this visit $\}$. For example, $\operatorname{PIP}(4,1,2, C, 3)$ and $\operatorname{PIP}(9,1,4, C, 8)$ are $C$ state PIP's that inherited from $\operatorname{PIP}(3,1,1, E, 1)$ and $\operatorname{PIP}(8,1,3, F, 7)$, respectively. Table I shows PIP's associated with three patients. Based on PIP data set, a patient-oriented folder can be constructed according to the $N$ value of PIP's and a problem-oriented folder constructed according to their $P$ value. The mutual relationship of these PIP's is shown in Fig. 1. If a physician wants to collect a complete set of a patient's medical information, he/she can combine the PIP's $S$ and $L$ values to link these histories. For example, there are 
TABLE I

Example List of Three Patients' Pip-Based Medical Records

\begin{tabular}{|c|c|c|c|c|}
\hline $\begin{array}{l}\text { Visitdate' }^{\prime} \\
\text { ProblemID }^{1}\end{array}$ & Date\# 1 & Datc \# 2 & Date\# 3 & Date\# 4 \\
\hline \multirow{3}{*}{ problem\#1 } & $\mathrm{PIP}(1.1 .1 . \mathrm{B} . \mathrm{NULL})^{2}$ & $\mathrm{PIP}(3,1,1, \mathrm{E}, 1)$ & & \\
\hline & $\operatorname{PIP}(1,2,1, B, N U L L)$ & $\operatorname{PIP}(2,2,1, F, 1)$ & $\mathrm{PIP}(4,2,1, \mathrm{E}, 2)$ & \\
\hline & $\operatorname{PIP}(1,3,1, B, N U L L)$ & $\operatorname{PIP}(3,3,1, F, 1)$ & $\operatorname{PIP}(5,3,1, F, 3)$ & $\operatorname{PIP}(6,3,1, F, 5)$ \\
\hline problem $\# 2$ & & $\operatorname{PIP}(4,1,2, \mathrm{C}, 3)$ & $\mathrm{PIP}(6,1,2, \mathrm{E}, 4)$ & \\
\hline problem\#3 & $\operatorname{PIP}(2,1,3, B, N U L L)$ & $\operatorname{PIP}(5,1,3, F, 2)$ & $\operatorname{PIP}(7,1,3, F, 5)$ & $\operatorname{PIP}(8,1,3, F, 7)$ \\
\hline \multirow[t]{2}{*}{ problem\#4 } & & & & $\operatorname{PIP}(9,1,4, \mathrm{C}, 8)$ \\
\hline & & $\operatorname{PIP}(3,2,4, \mathrm{~B}, \mathrm{NULL})$ & $\operatorname{PIP}(5,2,4, E, 3)$ & \\
\hline \multirow[t]{2}{*}{ problem\#5 } & & & & $\operatorname{PIP}(10,1,5, \mathrm{~B}, \mathrm{NULL})$ \\
\hline & $\operatorname{PIP}(2,3,5, B$, NULL $)$ & $\operatorname{PIP}(4,3,5, E, 2)$ & & \\
\hline
\end{tabular}

${ }^{1}$ the row represents the problem, the column represents the visiting date

${ }^{2}$ PIP(I, N, P, S, L) where I:PIPID. N:ChariNO, P:ProblemID, S:State, L:PreviousPIPID
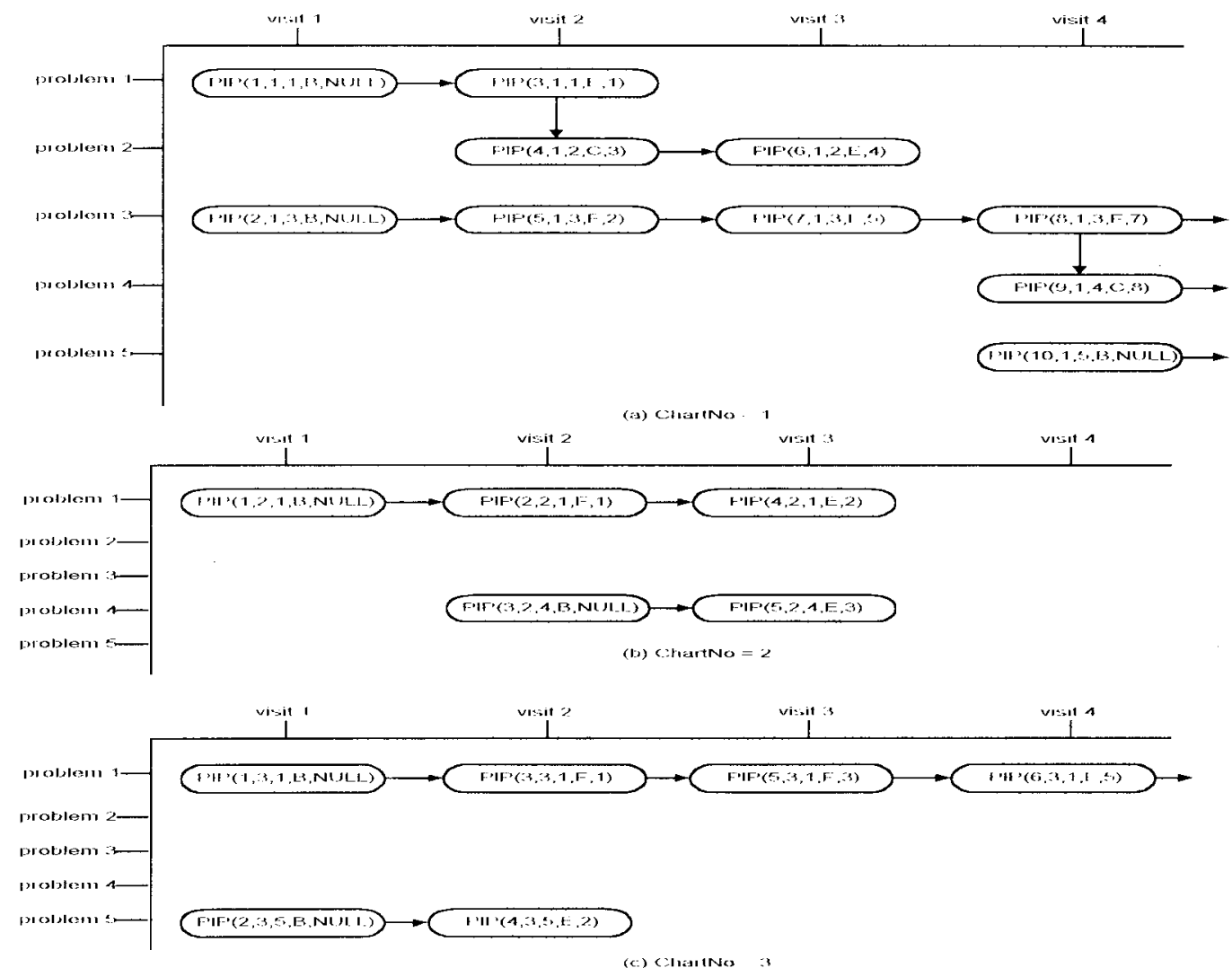

Fig. 1. Mutual relationship of patients PIP's in Table I.

ten PIP's [PIP( $1,1,1, B$, NULL $) \sim \operatorname{PIP}(10,1,5, B$, NULL $)]$ generated during four visits of the patient whose chart number is one in Fig. 1. Among the ten PIP's, there are four PIP's $[\operatorname{PIP}(2,1,3, B, \operatorname{NULL}), \operatorname{PIP}(5,1,3, F, 2), \operatorname{PIP}(7,1,3, F, 5)$, and $\operatorname{PIP}(8,1,3, F, 7)]$ pertaining to clinical problem 3 . That is, a PIP-based data set can help physicians obtain information not only on the diagnostic results of each visit, but also on the relationship between patient histories.

Besides, the PIP can manage and save any change of status or new information that emerges from the subjective description, objective description, assessment, and plan; these derivations are based on subjective, objective, assessment, and plan (SOAP) medical record methodology [35]. The subjective description $(S)$ refers to the description of a patient's chief complaint and the history of the disease problem. It is interpreted from the patient's point of view, and in this study, includes symptom code, duration, location, severity, description, and chief complaint. The objective description $(O)$ records the results of all measurements during the current visit and factual plan results as noted by the physician during the previous visit concerning the same problem. In this part, physical examination results, laboratory data, and the diagnostic plan conclusions are summarized in the fields of item, location, finding, sign-code, and description. The assessment information, part A, records the physician's diagnosis and a description of the disease problem based on the information in part $S$ and part $O$. It is expressed with the problem ID and an assessment description. The plan information, part $P$, refers to the diagnostic and therapeutic plans made by the physician specifically addressing the patient's problem. 


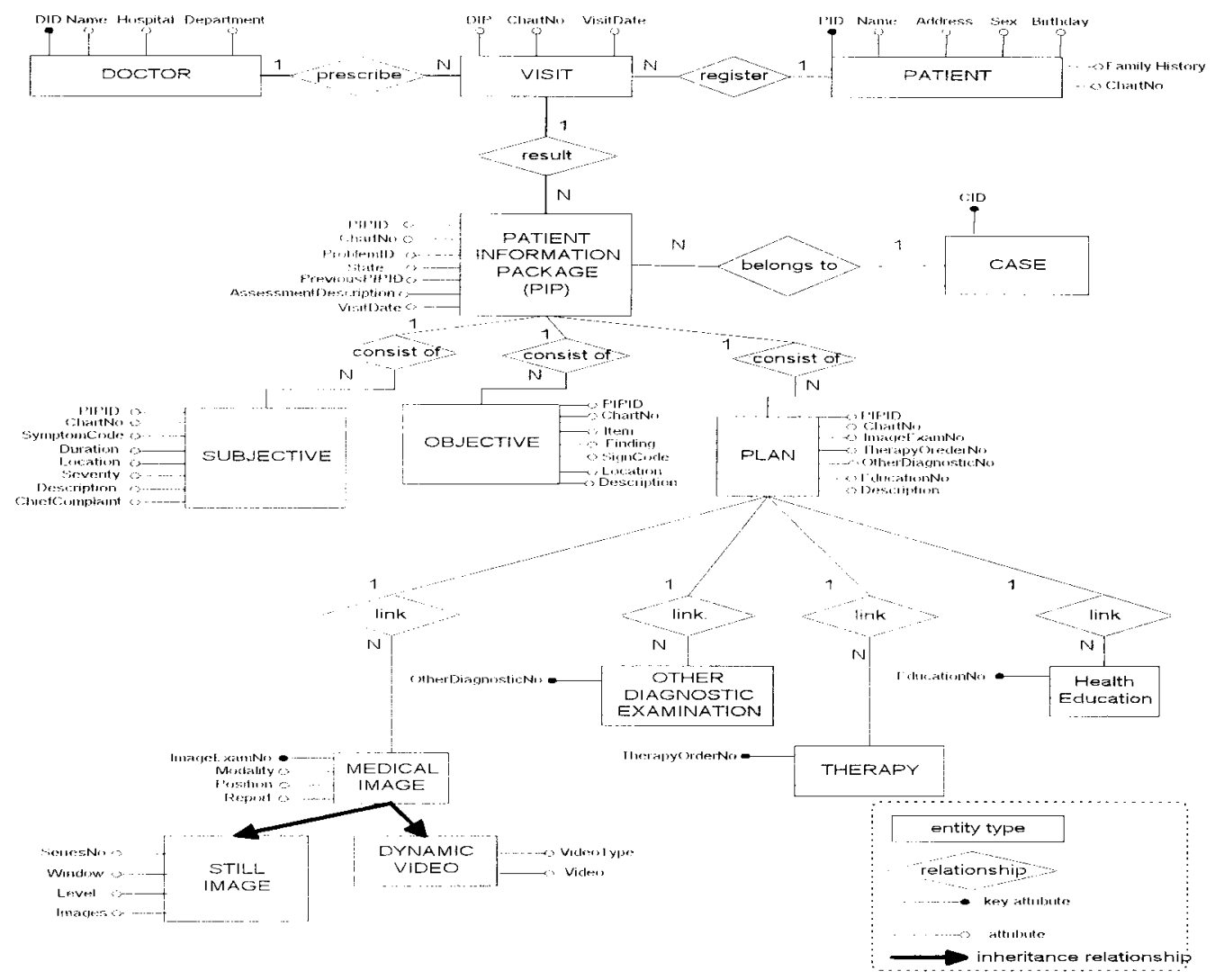

Fig. 2. Entity-relation (ER) diagram of PIP-based medical database.

This section includes all descriptions of medication, treatment, laboratory tests, diagnostic plans (such as X-ray studies), and patient education.

As shown in Fig. 2, an enhanced entity-relationship diagram [32]-[34] can be used to model the scenarios of patient medical information. DOCTOR entity, PATIENT entity, VISIT entity, and $C A S E$ entity are the sets of attributes of physicians, patients, patient's visit, and clinical case, respectively. Because a patient can register and a physician can prescribe more than one clinical visit, they introduce a one-to-many relationship to VISIT entity. Every PIP is emerged according to one problem diagnosed by the physician. If there are many problems concerned during one patient's visit, the physician will prescribe many PIP's. Therefore, there is a one-to-many relationship between VISIT entity and PIP entity. Because every problem has only one final assessment, the ProblemID and AssessmentDescription that result from the assessment of SOAP are combined with PIP entity. Thus, the entity type PIP consists of attributes PIPID, ChartNo, ProblemID, State, PreviousPIPID, AssessmentDescription, and Visitdate. It has a composite primary key made of ChartNo and PIPID. Except for assessment information, the other parts of the SOAP record methodology may generate distinct entities. The entities of SUBJECTIVE, OBJECTIVE, and PLAN all have a many-to-one relationship with $P I P$ entity. Because plans are realized by means of diagnostic, therapeutic, and educational prescriptions, there may be more than one order prescribed by the physician. PLAN entity has a one-to-many relationship with the entities of MEDICAL-
IMAGE, OTHER-DIAGNOSTIC-EXAMINATION, THERAPY, and HEALTH-EDUCATION.

In the manipulation of medical images, the categorization is based on image modality. The entities of MEDICAL-IMAGE, STILL-IMAGE, and DYNAMIC-VIDEO form the inheritance lattice shown in Fig. 2. MEDICAL-IMAGE entity is a superclass of the entities of STILL-IMAGE and DYNAMICVIDEO. X-ray, CT, and MR images are part of STILL-IMAGE entity. STILL-IMAGE entity has the attributes of SeriesNo, Images, Window, and Level. The SeriesNo attribute is used to indicate the sequence of the set of images, and the Images attribute is used to store the images that belong to that series. In addition, STILL-IMAGE entity has the attributes of ImageExamNo, Modality, Position, and Report that inherit from the superclass entity of MEDICAL-IMAGE. Similarly, in addition to attributes inherited from MEDICAL-IMAGE entity, $D Y N A M I C$-VIDEO entity has the attributes of VideoType and Video.

\section{Database Implementation}

It is noteworthy that, as in Fig. 2, the medical record is a form of multimedia. An important point in system design is how to build a medical information database system to manage heterogeneous data. Although the relational database provides a set of powerful tools to manipulate data, its template of predefined data type limits its ability to manage large objects. Conventionally, a relational database management system manages large objects with a binary large object (BLOB) field. However, the huge data size of the records may 


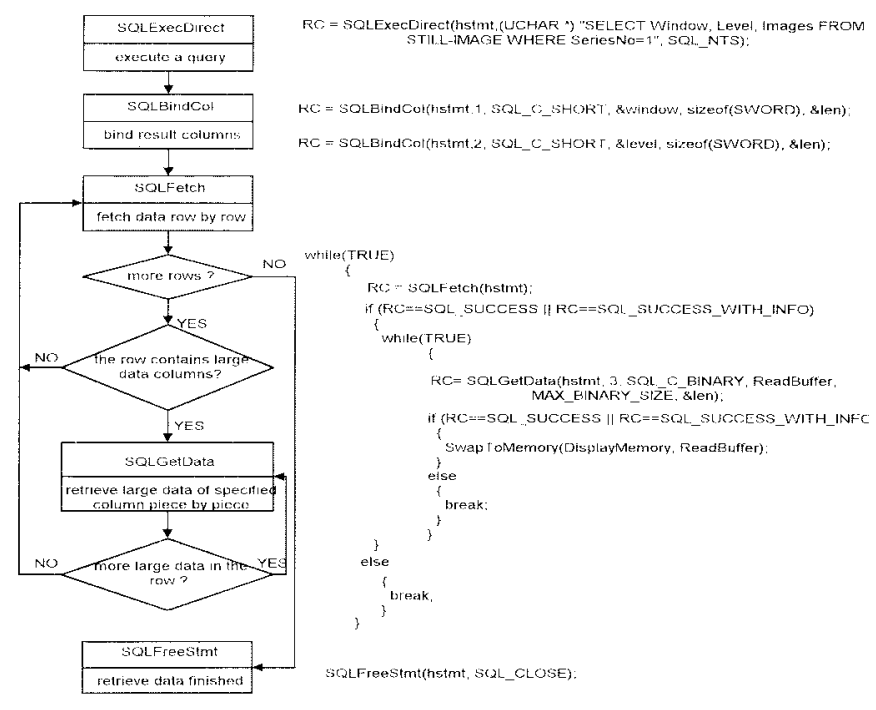

Fig. 3. Program flow for retrieving multimedia medical data from database.

greatly reduce the hit ratio of the database system cache. This forces the system developer to put large objects in a separate file that is always handled by a different piece of software. This kind of management method may introduce difficulties in data consistency and maintenance. The complexity of data archiving may also perplex system administrators and developers. One solution to overcome this limitation is to expand the data type for external files in the database system. This can improve the consistency and simplify the maintenance of the database system and, furthermore, provide a unified access mechanism for all types of data.

After performing a survey of current state-of-thecommercial-art database systems, we choose DBMaker (CaseMaker Inc., USA) to manage the medical records. DBMaker is an object-relational database management system (ORDBMS). It provides two data types, BLOB (binary large object) and FILE (file object), through which large image objects can be handled directly with the standard structured query language (SQL). When an attribute is defined as BLOB or FILE type, it is regarded as a stream sequence and managed by the DBMS using stream operations. When the size of a BLOB-type attribute is greater than $4 \mathrm{~K}$ bytes, it is stored by frame in a system-default file (called a BLOB file). Otherwise, it is stored in the database itself. A FILE-type attribute is stored in a unique file created by the database itself. The implementation of BLOB and FILE data types can assure data consistency and simplify database management.

In our implementation, the attributes of Report, ChiefComplaint, and Description are defined as BLOB type. The attributes of Video and Images are defined as FILE type. Other attributes, such as PIPID, Name, ImageExamNo, and so on, are defined similarly to the relational database. Through implementation of BLOB and FILE, all patient medical records can be systematically integrated and managed by the same database management system. More importantly, they can be uniformly manipulated in SQL queries. For example, we create the STILL-IMAGE table that includes the fields of SeriesNo (numeral type), Window (numeral type), Level (numeral type), and Images (File type); we try to fetch the columns Window, Level, and Images from SeriesNo $=1$ in the STILL-IMAGE table. The steps for open database connectivity (ODBC) command are shown in Fig. 3. After a query statement is executed by SQLExecDirect command, we use the bind method to get the values of the Window and Level columns and use SQLGetData to get the value contained in the Images column. The bind method uses SQLBindCol to bind a local buffer for the column. During SQLFetch, the column data are automatically stored in the bound buffer. When we need to retrieve large image data, we call SQLGetData command to get one piece of the image file in the unbound column in the current row.

In addition to data integration, speed of data retrieval is also a factor that affects the performance of the telemedicine system. In this paper, a three-layer hierarchical database is created; the three layers consist of main database, long-term database, and local database. The main database stores medical information concerning patients who have visited within recent months. After this period, the data are moved to a long-term database. Then the long-term database server packs the image data according to time of creation and manages it in the DICOM media storage directory (DICOMDIR) format, which is introduced by the American College of Radiology and the National Electrical Manufacturers Association (ACR/NEMA) to store DICOM-formatted medical images in permanent media [36].

The local database provides a short-term storage location for the medical records of patients currently visiting. It functions to reduce workload of the database server and traffic of the network. In order to prepare the most frequently used data, the PREFETCH mechanism, which works to reduce the data accessing time, is incorporated into the local database installed in the medical center. During teleconsultation, the PREFETCH precedes the diagnosis and accesses medical records according to the schedule. In telediagnosis, the medical records must also be prefetched if the diagnosis report has not yet been completed. Moreover, the REFRESH mechanism is also incorporated in the local database at the rural site to maintain acceptable communication reliability. It stores the medical records of newly visiting patients in the local database and forward these records when the communication channel has been successfully connected. Thus, it can avoid data loss caused by failure of the communication channel.

\section{User Interface Design}

The PIP-based medical database provides a unified dataaccess interface to present patient-oriented or problem-oriented medical records. In our implementation, it is implemented with the object relational database management system and can be accessed through the ODBC interface (as shown in Fig. 4). Software development for data access is needed only one time for all different applications. This achieves software reuse and reduces the effort required for system development. System developers can pay more attention to the design of a user interface for specific purposes in different applications.

User acceptance depends on the quality of the user interface [37]-[38]. In order to satisfy the requirements for different 


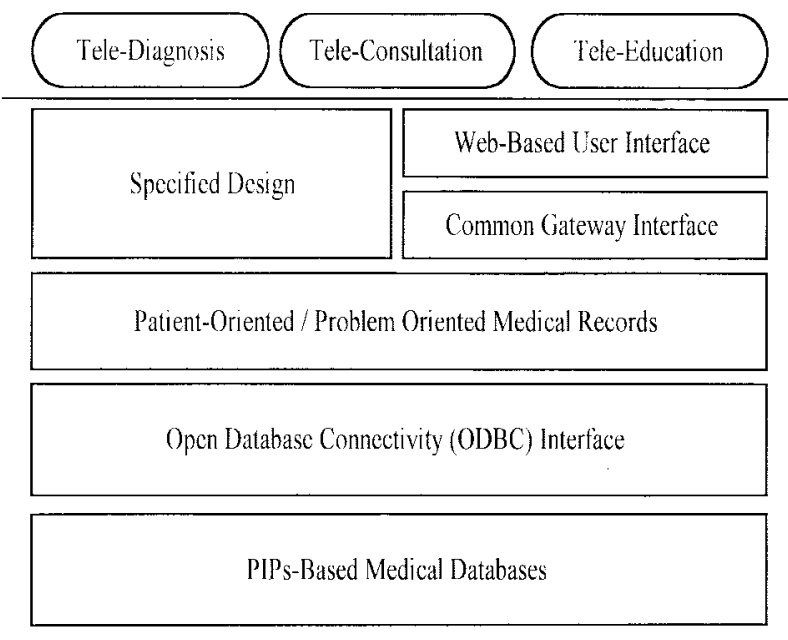

Fig. 4. Function diagram of our telemedicine system.

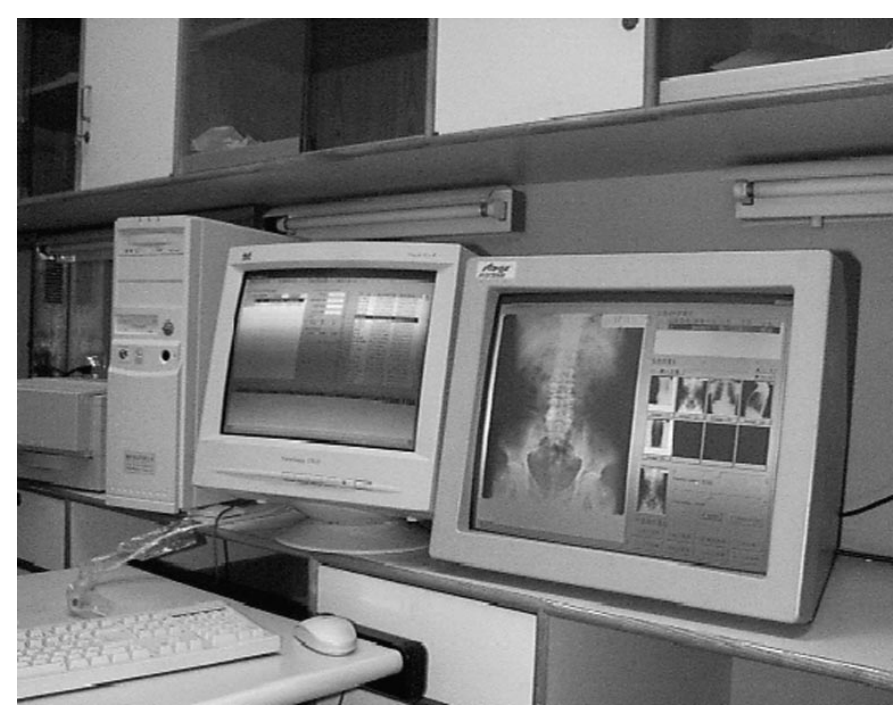

Fig. 5. Dual-monitors user interface for high-quality image display. Right side is a high-illuminate monitor; left side is an SVGA monitor.

types of medical services, we develop two kinds of user interface in this study. For high-quality data display, the system is equipped with two monitors (Fig. 5). An SVGA monitor is used to present dynamic patient videos and other data; another high-resolution and high-illumination monitor is used to display still images. The maximal spatial resolution of a still image can be up to $2048 \times 2487$ pixels to accommodate the largest size of an x-ray film, and the gray-level depth can be up to 12 bits per pixel. In order to display the image appropriately, the window value of an image is initially set within the range from 10 to $90 \%$ of the accumulated histogram of the image. The level is set to the medium value of this range. The computation of histogram, window, and level mapping of an image are shown in Fig. 6. Several software functions (e.g., zoom in/out, vertical/horizontal flip, 90/180/270 degree rotation, and window/level adjustment) are implemented to process and display medical images on the dual-monitor viewing station.

For convenient usage and feasibility in a multiplatform system, a web-based interface is used to integrate and display the categorized information. In order to display the medical records with a web browser, a conventional web browser must be enhanced with the ability to convert the special image format, display medical images rapidly, and provide imageprocessing tools for clinical practice. In our implementation, a common gateway interface (CGI) program is developed to access the medical records with the ODBC interface, convert medical images in the DICOM 3.0 format to the joint-picture-expert group (JPEG) format, and compress the images according to user requirements. Moreover, a plug-in program is added to the browser for image processing. The software architecture of the web-based user interface is shown in Fig. 7. After identifying a logged-on user, the CGI program is invoked either to link other homepages or access the medical records from the database. If the required data contain images, the CGI program can convert 12-bit images to 8-bit ones or downsample the images to one-fourth size for speeding the image transformation. The plug-in program embedded in the web browser is invoked after the user selects an image of interest. This program will download the original image in the DICOM format from the database server and provide image-processing tools for the user.

\section{APPLICATIONS}

\section{A. NTUH-Chinshan Telemedicine System}

Chinshan is a small village in the most northern part of Taiwan. The village has 17000 residents and is connected to the outside world by only two roads. The only healthcare service in Chinshan is a group practice center affiliated with NTUH. Therefore a telemedicine system is introduced to 1) provide teleconsultation, 2) provide follow-up service for patients suffering from chronic illnesses, and 3) facilitate the training of clinicians and medical students working in this rural site.

The system has been in operation since January 1997 [39]. The telemedicine system was used only on Tuesday and Friday afternoons during the first six months. The architecture of the telemedicine system was shown in Fig. 8, and the hardware devices were listed in Table II. In this project, the communication network between NTUH and Chinshan was DS1 private line (T1). It was divided into two bandwidths for video conferencing and data transmission. A teleconferencing system (GPT) could capture physicians' interactive conversations for teleconsultation and tele-education. Transmission data from Chinshan included chief complaint, history of illness, results of physical examination, x-ray images, ultrasonic, and CCD dermatology videos. X-ray images were a kind of STILLIMAGE data type. They were digitized by a high-resolution laser scanner. Ultrasonic and CCD dermatology videos were kinds of DYNAMIC-VIDEO type. A real-time motion-pictureexpert group 1 (MPEG1) was needed for digitization.

Until August 1997, 31.3\% of 809 visiting patients were more than 65 years old. This showed that the elderly residents of this rural location used the telemedicine system most frequently. The most commonly used service was radiology consultation $(15 \%)$ due to a lack of radiologists at the rural site. The second was ultrasonic real-time consultation involving liver disease (about 13.4\%). Chronic liver disease was one 

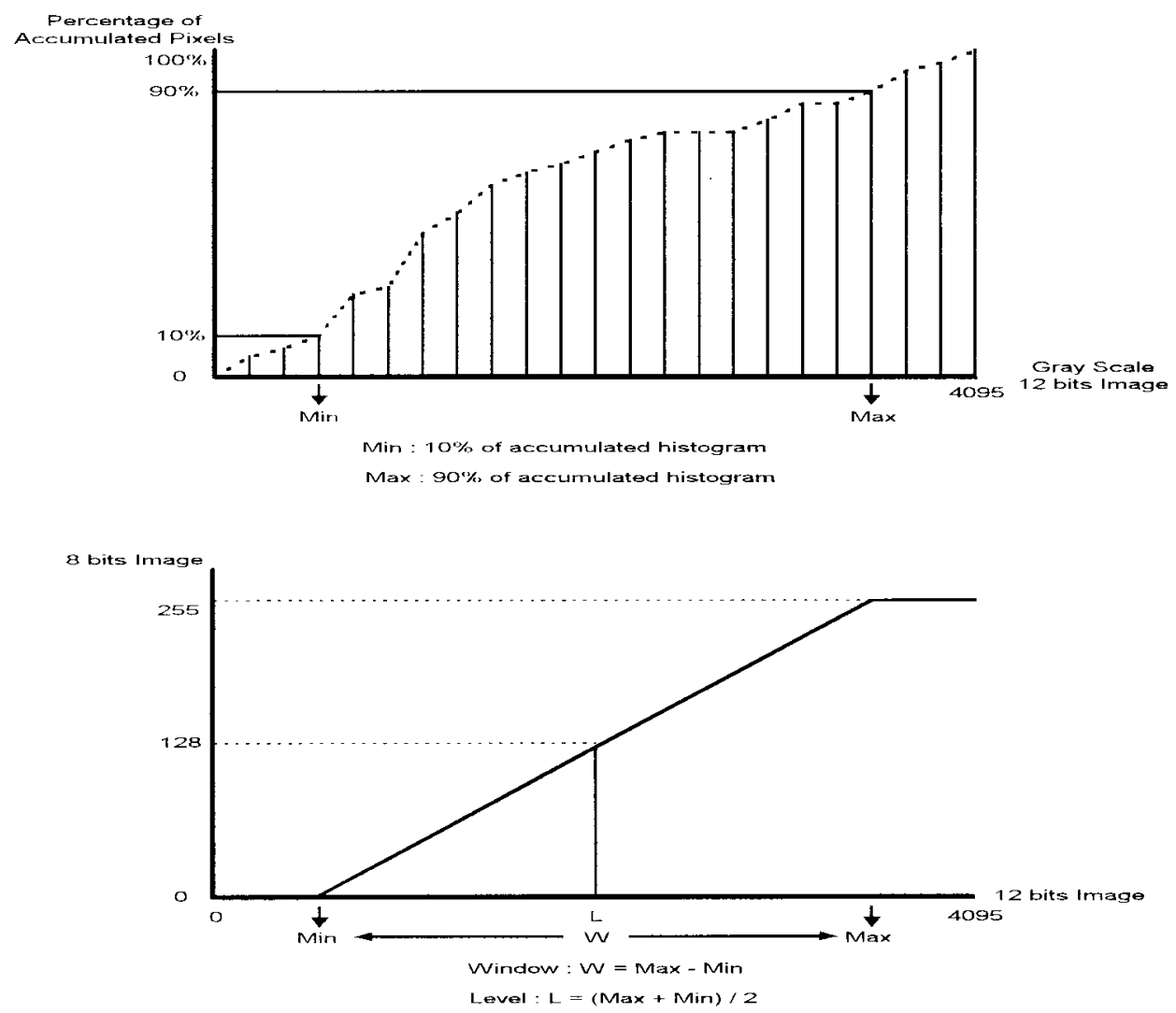

Fig. 6. Concept of optimal window/level adjustment.

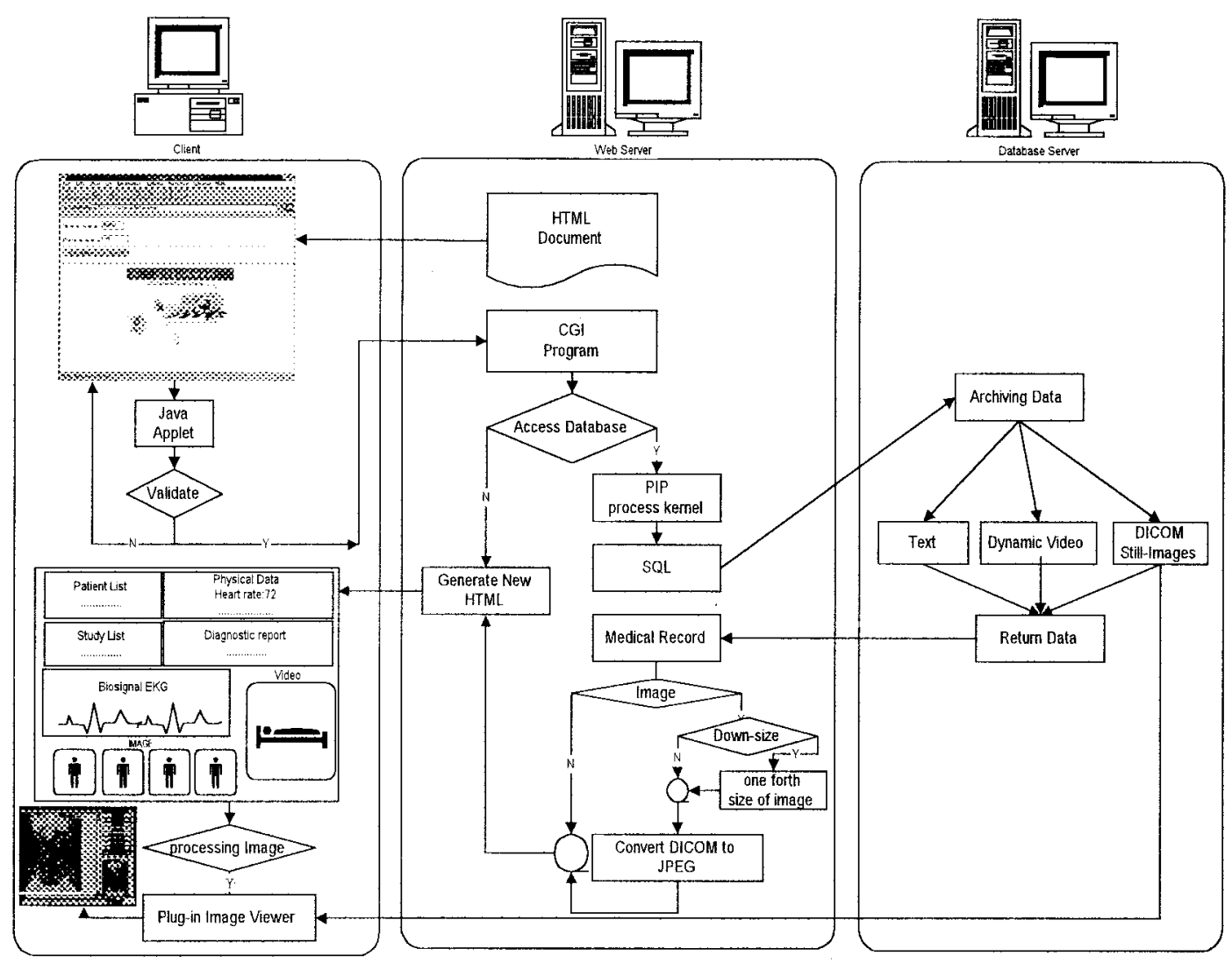

Fig. 7. Software architecture of web-based telemedicine system for tele-education. 


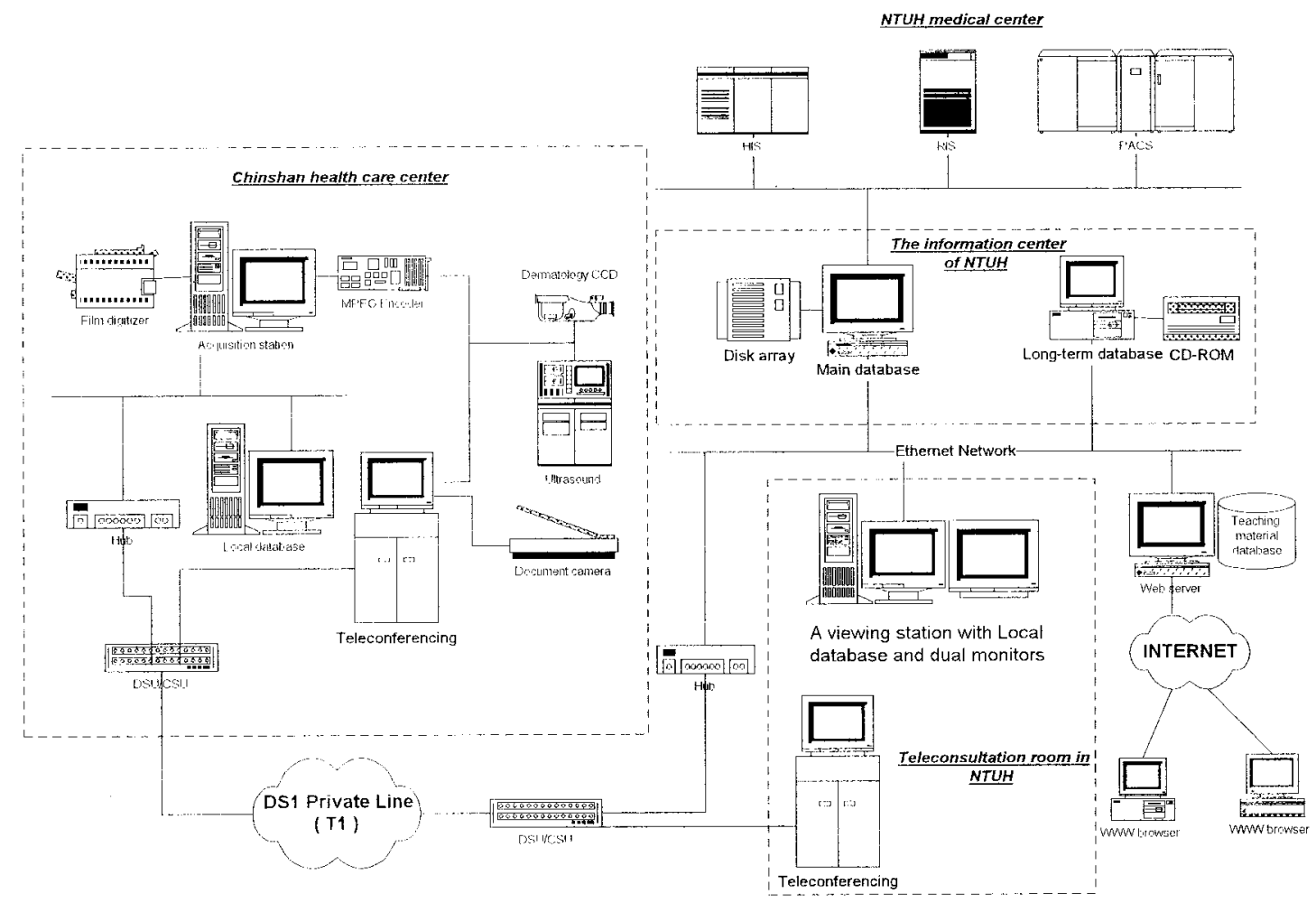

Fig. 8. Architecture of telemedicine between NTUH and Chinshan healthcare center. The left side is the setting up at the Chinshan healthcare center; and the right side is setting up at NTUH.

TABLE II

Specification of the Hardware Device of the Telemedicine System

\begin{tabular}{|c|c|c|}
\hline Device & Specificition & Quantity \\
\hline Laser Film Digilizcr & 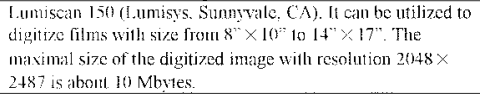 & 1 \\
\hline MPEG Encoder/Decoder & $\begin{array}{l}\text { VITEC MPEGI RT - } 1 \text { li. } \\
\text { Video clip MPEG / MPEG tool box } \\
\text { bit ratc (video): } 380 \mathrm{Kbps} \sim 5 \text { Mbps } \\
\text { bit ralc (audio): } 32 \mathrm{Kbps} \sim 38+\mathrm{Kbps} \text {. }\end{array}$ & 1 \\
\hline $\begin{array}{l}\text { High-Resolution CCD } \\
\text { Camcril }\end{array}$ & 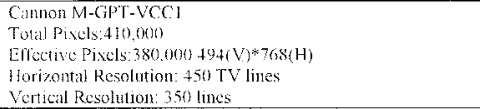 & 1 \\
\hline Doclumentalion Machine & Camunin RE-650 MK II & 2 \\
\hline Tele-Conferencing Syslem & $\begin{array}{l}\text { GP Focus } 100 \text { bi-directional real-tine fele-conferencing } \\
\text { sistem. }\end{array}$ & 2 \\
\hline $\begin{array}{l}\text { High-Resolution and High- } \\
\text { Illuntination Mouitor }\end{array}$ & 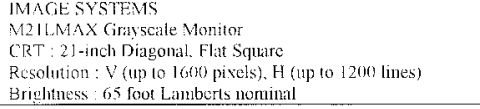 & 4 \\
\hline Viewing Station & PC hild PENTLMM-200.64 M byles RAM & 6 \\
\hline High-Capacity Disk Array & $\begin{array}{l}\text { PROWARE DISKRAID 486DX } \\
\text { RAID processor : } 4860 \mathrm{DX} \\
\text { Support RAID 0.135 } \\
\text { 3 SCSI Channels } \\
\text { Hol Swap Disk Bays*8 } \\
\text { Hal Swap l'ower Supply*2 } \\
\text { + GiB*5. }\end{array}$ & 1 \\
\hline bocal Area Network & ETHERNET : $10 \mathrm{Mbits} / \mathrm{s}$ & - \\
\hline Wide Areál Nelwork & DSI-private line $(T-1): 1.544$ Mbis $/ \mathrm{s}$ & - \\
\hline
\end{tabular}

of the major causes of mortality and morbidity in Taiwan, and ultrasound was an important tool for screening and follow up.

To assess the responses of patients and doctors to the system, we conducted a questionnaire survey. Among the patients, $86.6 \%$ were very satisfied with the consultant doctor in the telemedicine system. Among the physicians, the on-site physicians were more satisfied with the telemedicine system than those at NTUH. Among all cases of teleconsultation, $15 \%$ of diagnoses were modified by specialists at NTUH. This result showed that the telemedicine system was a useful way to improve the quality of medical services at a rural site.

\section{B. Web-Based Tele-Education}

A web-based course material editing [Fig. 9(a)] and case presentation [Fig. 9(b)] system was created by the National Taiwan University Medical Center [40]. Tele-education under a web-based environment was comparable with medical education in a face-to-face situation. The survey subjects were 40 fifth-grade male medical students in the medical school, and the results of such a survey are shown in Fig. 10. Results show that more than $60.9 \%$ students think it was easy to access references. There were 58.1 and $43.7 \%$ of students that were satisfied with case presentation and image display, respectively. It showed that a web-based educational environment was relatively convenient for accessing references and presenting cases. Due to the web-based environment lack of face-to-face communication, it was less convenient than a conventional one for the instructor to discuss with other students (as shown in Fig. 10). Thus, how to combine the web-based tele-education system with teleconferencing was the essential direction for work in the near future.

\section{CONClusions AND AREAS OF FurTher EFFORT}

This paper describes a telemedicine system used to provide medical services to a rural healthcare center. Three operational modes of the telemedicine system are explored through the system developed. In order to fulfill the requirements of medical practice, we define a PIP that functions as a databaseprocessing element encapsulating medical information obtained during one patient visit. A PIP-based data structure can reduce the complexity of accessing medical information. In this study, we also integrate multimedia patient information 


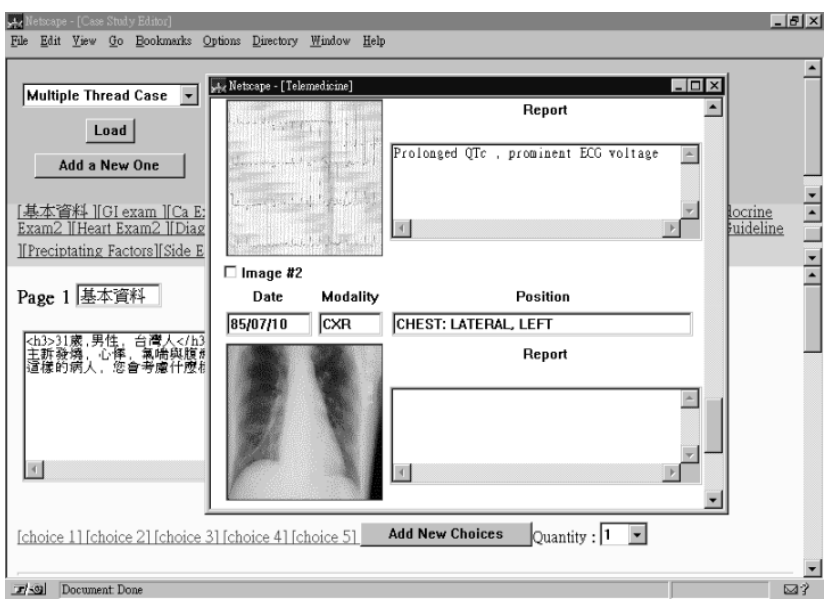

(a)

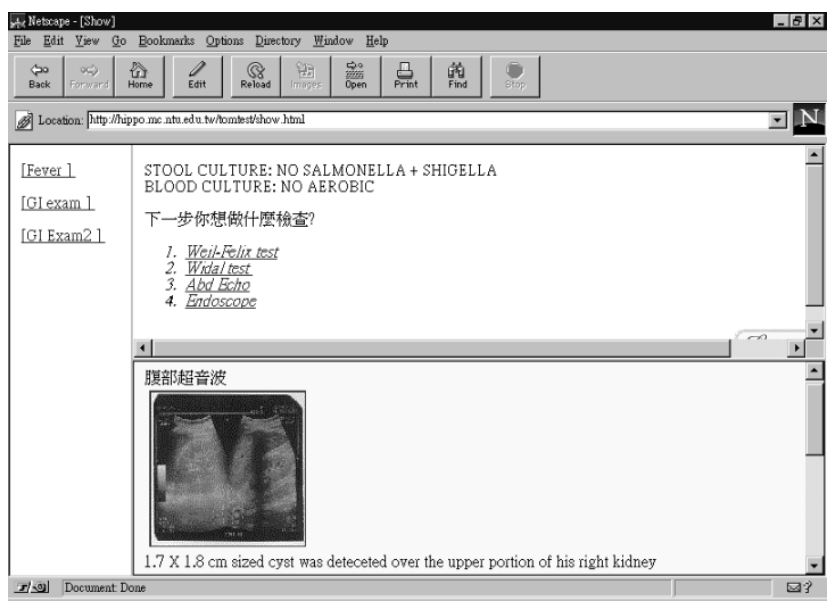

(b)

Fig. 9. Web-based tele-education system: (a) User interface of course material editing. (b) User interface of case presentation.

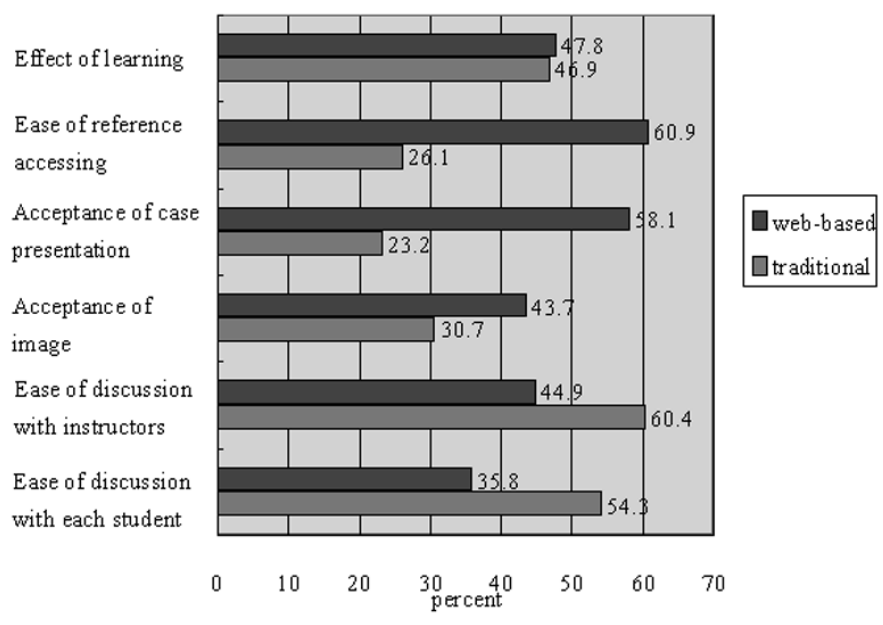

Fig. 10. Results of comparing web-based education to traditional education

within the same database system and provide two kinds of user interfaces for different medical service purposes.

The medical services provided by the telemedicine system at the rural site is eagerly needed by the elderly. The system allows the elderly to avoid traveling a long distance to get better care. Evaluation results show that the telemedicine system is relatively feasible in the case of teleradiology. Telemedicine has shown the capability not only to improve the quality of healthcare, but also to increase the opportunity of continuing education for physicians at a rural site. According to the results of the survey, the WWW environment's features of multimedia and hyperlinking made the web-based browser suitable for displaying medical teaching materials.

Based on the system developed, there are many other aspects that can be explored in the future. One is to add a datamining technique to the system [39]. This could allow the formulation of diagnostic behaviors and build a knowledge base to assist diagnosis and medical teaching. The other is to incorporate image compression technique to speed image transmission [40]. These advances may help researchers to not only explore the knowledge of medical behavior, but also expand the feasibility of the telemedicine system.

\section{ACKNOWLEDGMENT}

The authors would like to thank Dr. C.-Y. Chen, Dr. Y.-C. Chang, Dr. Y.-L. Lui, and Dr. F.-R. Guo for their enthusiastic involvement in this research.

\section{REFERENCES}

[1] B. H. Guze, R. Estep, and C. Fisher, "Telemedicine: A review of its use and a proposal for application in psychiatric consultation," Med. Inform., vol. 20, no. 1, pp. 1-18, 1995.

[2] J. E. Cabral, Jr. and Y. Kim, "Multimedia systems for telemedicine and their communications requirements," IEEE Commun. Mag., July 1996, pp. 20-27.

[3] T. Paakkala, J. Aalto, V. Kähärä, and S. Seppänen, "Diagnostic performance of a teleradiology system in primary health care," Comput. Methods Programs Biomed., vol. 36, pp. 157-160, 1991.

[4] J. Viitanen, T. Sund, E. Rinde, J. Stoermer, M. Kormano, J. Heinila, J. Yliaho, and J. Ahonen, "Nordic teleradiology development," Comput. Methods Programs Biomed., vol. 37, pp. 273-277, 1992.

[5] H. K. Huang, "Teleradiology technologies and some service models," Comput. Med. Imag. Graph., vol. 20, no. 2, pp. 59-68, 1996.

[6] O. Ratib, Y. Ligier, and J. R. Scherrer, "Digital image management and communication in medicine," Comput. Med. Imag. Graph., vol. 18, no. 2, pp. 73-84, 1994.

[7] H. K. Huang et al., "Implementation of a large-scale picture archiving and communication system," Comput. Med. Imag. Graph., vol. 17, no. 1, pp. 1-11, 1993.

[8] D. F. Leotta and Y. Kim, "Requirements for picture archiving and communications," IEEE Eng. Med. Biol. Mag. , pp. 62-69, Mar. 1993.

[9] H. K. Huang, W. K. Wong, S. L. Lou, and B. K. Stewart, "Architecture of a comprehensive radiologic imaging network," IEEE J. Select. Areas Commun., vol. 10, pp. 1188-1196, Sept. 1992.

[10] W. J. Chimiak, "The digital radiology environment," IEEE J. Select. Areas Commun., vol. 10, pp. 1133-1144, Sept. 1992.

[11] S. T. Treves, E. S. Hashem, B. A. Majmudar, K. Mitchell, and D. J. Michaud, "Multimedia communications in medical imaging," IEEE J. Select. Areas Commun., vol. 10, pp. 1121-1132, Sept. 1992.

[12] S. L. Lou, J. Wang, M. Moskowitz, T. Bazzill, and H. K. Huang, "Methods of automatically acquiring images from digital medical systems," Comput. Med. Imag. Graph., vol. 19, no. 4, pp. 369-376, 1995.

[13] Digital Imaging and Communications in Medicine (DICOM) Version 3.0, Amer. College Radiologists/Nat. Elect. Manufacturers Assoc., 1993.

[14] G. Bucci, R. Detti, S. Nativi, and V. Pasqui "Loosely coupled workstations in a radiological image information system," Future Generation Comput. Syst., vol. 8, pp. 31-42, 1992.

[15] S. K. Mun, M. Freedman, and R. Kapur, "Image Management and communications for radiology," IEEE Eng. Med. Biol. Mag., pp. 70-80, Mar. 1993.

[16] G. Hartviksen, S. Akselsen, A. K. Eidsvik, S. Pedersen, and E. Rinde, "Toward a general purpose, scaleable workstation for remote medical consultations. Experiences from use of VIDA-a still image system for the provision of low-cost telemedicine," Med. Inform., vol. 20, no. 1, pp. 19-33, 1995. 
[17] H. Handels, C. Busch, J. Encarnaçao C. Hahn, V. K $\square$ hn, J. Miehe, S. I. Pöppl, E. Rinast, C. Roßmanith, F. Seibert, and A. Will, "KAMEDIN: A telemedicine system for computer supported cooperative work and remote image analysis in radiology," Comput. Methods Programs Biomed., vol. 52, pp. 175-183, 1997 .

[18] F. R. Bartsch, M. Gerneth, and R. Schosser, "Videoconference as a tool for European inter-hospital consultations in radiology," in Proc. SPIE, 1977, pp. 62-67.

[19] S. J. Dwyer, III, et al., "Teleradiology using switched dialup networks," IEEE J. Select. Areas Commun., vol. 10, pp. 1161-1172, Sept. 1992.

[20] L. Orozco-Barbosa, A. Karmouch, N. D. Georganas, and M. Goldberg, "A multimedia interhospital communications system for medical consultations," IEEE J. Select. Areas Commun., vol. 10, pp. 1145-1157, Sept. 1992.

[21] K. Chipman, P. Holzworth, J. Loop, N. Ransom, D. Spears, and B. Thompson, "Medical applications in a B-ISDN field trial," IEEE $J$. Select. Areas Commun., vol. 10, pp. 1173-1187, Sept. 1992.

[22] H. K. Huang, R. L. Arenson, S.-L. Lou, A. W. K. Wong, K. P. Andriole, T. M. Bazzill, and D. Avrin, "Multimedia in the radiology environment: Current concept," Comput. Med. Imag. Graph., vol. 18, no. 1, pp. 1-10, 1994.

[23] G. F. Egan and Z.-Q. Liu, "Computers and networks in medical and healthcare systems," Comput. Biol. Med., vol. 25, no. 3, pp. 355-365, 1995.

[24] T. Kitanosono, Y. Kurashita, M. Honda, T. Hishida, H. Konishi, M. Mizuno, and M. Anzai, "The use of multimedia in patient care," Comput. Methods Programs Biomed., vol. 37, pp. 259-263, 1992.

[25] M. H. Williams and J. Hu, "Making heterogeneous medical database interoperable," Comput. Methods Programs Biomed., vol. 43, pp. 275-281, 1994.

[26] S. T. C. Wong and H. K. Huang "A hospital integrated framework for multimodality image base management," IEEE Trans. Syst., Man, Cybern. A, vol. 26, pp. 455-469, July 1996.

[27] R. K. Taira, B. K. Stewart, and U. Sinha, "PACS database architecture and design," Comput. Med. Imag. Graph., vol. 15, no. 3, pp. 171-176, 1991.

[28] S. Badaoui, V. Chameroy, and F. Aubry, "A database manager of biomedical images," Med. Inform., vol. 18, no. 1, pp. 23-33, 1993.

[29] F. Pinciroli, C. Combi, and G. Pozzi, "ARCADIA: A system for the integration of angiocardiographic data and images by an object-oriented DBMS," Comput. Biomed. Res., vol. 28, pp. 5-23, 1995.

[30] A. Karmouch, "Multimedia distributed cooperative system," Comput. Commun., vol. 16, pp. 568-580, Sept. 1993.

[31] K. Kurisu, Y. Hishikawa, M. Izumi, M. Taniguchi, N. Kamikonya, and N. Nakao, "Personal computer-based small PACS for radiotherapy analog image filing system for radiotherapy," Comput. Methods Programs Biomed., vol. 43, pp. 71-73, 1994.

[32] P. Chen, "The entity-relationship model-toward a unified view of data," ACM Trans. Database Syst., vol. 1, pp. 9-36, 1976.

[33] A. Silberschatz, H. F. Korth, and S. Sudarshan, Database System Concepts, 3rd ed. Singapore: McGraw-Hill, 1997.

[34] T. Connolly, C. Begg, and A. Strachan, Database systems-A Practical Approach to Design, Implementation and Management. Wokingham, U.K.: Addison-Wesley, 1996.

[35] R. E. Rakel, Textbook of Family Practice, 5th ed. Philadelphia, PA: Saunders, 1995.

[36] Digital Imaging and Communications in Medicine (DICOM) Version 3.0, Amer. College Radiologists/Nat. Elect. Manufacturers Assoc., 1993.

[37] H. U. Lemke, G. Faulkner, and M. Krauss, "Development toward multimedia medical workstations," Comput. Med. Imag. Graph., vol. 18, no. 2, pp. 67-71, 1994.

[38] E. J. Gömez, F. del Pozo, J. A. Quiles, M. T. Arredondo, H. Rahms, M. Sanz, and P. Cano, "A telemedicine system for remote cooperative medical imaging diagnosis," Comput. Methods Programs Biomed., vol 49 , pp. 37-48, 1996.

[39] C. T. Liu, C. C. Lin, J. M. Wong, S. K. Chiou, R. S. Chen, J. H. Chen, S. M. Hou, and T. Y. Tai, "Design and evaluation of a telediagnosis system," Biomed. Eng. Applicat., Basis Commun., vol. 9, pp. 52-60, Apr. 1997.

[40] H. S. Chen et al., "Integrated medical informatics with small group teaching in medical education," Int. J. Med. Inform., to be published.

[41] M. S. Chen, J. Han, and P. S. Yu, "Data Mining: An overview from a database perspective," IEEE Trans. Knowl. Data Eng., vol. 8, pp. 866-883, Dec. 1996

[42] W. Y. Kim, P. T. Balsara, D. T. Harper, and J. W. Park, "Hierarchy embedded differential image for progressive transmission using lossless compression," IEEE Trans. Circuit Syst. Video Technol., vol. 5, pp. 1-13, Feb. 1995.
Chung-Chih Lin (S'94) received the B.S. and M.S. degrees in biomedical engineering from Chung Yuan University, Chungli, Taiwan, R.O.C., in 1992 and 1994, respectively. He is currently pursuing the Ph.D. degree in electrical engineering at the National Taiwan University, Taipei.

His research interests include medical informatics, WWW application, multimedia, database, artificial neural network for biomedical application, and data compression.

Jeng-Ren Duann (S'91) received the B.S. and M.S. degrees in biomedical engineering from Chung Yuan University, Chungli, Taiwan, R.O.C., in 1990 and 1992, respectively. He is currently pursuing the Ph.D. at Chung Yuan University.

His current research interests include medical signal and image processing, medical informatics, and biosystem (e.g., heart) modeling.

Chien-Tsai Liu received the M.S. degree in electronic engineering from the National Taiwan Institute of Technology in 1986 and the Ph.D. degree in computer science from the University of Pittsburgh, Pittsburgh, PA, in 1994.

$\mathrm{He}$ is currently an Associate Professor and Director of the Department of Information Systems, Wanfang Hospital, Taipei Medical College, Taipei, Taiwan, R.O.C.. His research interests include data modeling, medical database systems, design and evaluation of telemedicine systems, and software development methodology, especially for hospital information systems.

Heng-Shuen Chen received the M.D. degree from the National Taiwan University, Taipei, Taiwan, R.O.C., in 1985 . He is currently pursuing the $\mathrm{Ph} . \mathrm{D}$. degree in electrical engineering at the National Taiwan University.

He received Residency and Fellowship training from 1987 to 1992 and became a faculty member in 1992 with the Family Medicine Department, National Taiwan University Hospital. Currently, he is also a Senior Lecturer and the Director of Medical Informatics Program in Department of Medical Informatics, College of Medicine, National Taiwan University. His research interests include, but are not limited to, computer-assisted learning, telemedicine, distance education, preventive medicine, and computerized medical record.

Jenn-Lung Su (S'84-M'88) received the B.S. (honors) degree in biomedical engineering from Chung Yuan University, Chungli, Taiwan, R.O.C., in 1977 and the M.S. and Ph.D. degrees in bioengineering in 1985 and 1988, respectively, from The University of Illinois, Chicago.

He was Chairman of the Department of Biomedical Engineering, Chung Yuan University, from 1989 to 1995 . Since 1988, he has been an Associate Professor at Chung Yuan University. He has also served as the Chief Editor of the Chinese Journal of Medical and Biological Engineering since 1996. His current research interests include medical imaging, medical informatics, and electromagnetic interaction with biological systems.

Jyh-Horng Chen (S'89-M'91) was born in 1960. He received the B.S. degree from the National Taiwan University (NTUEE), Taipei, Taiwan, R.O.C., in 1982 , the M.S. degree in biomedical engineering from the National Yang Ming Medical College, and the Ph.D. degree in biomedical engineering from the University of California at Berkeley/University of California at San Francisco Joint Bioengineering Program.

He has been with the faculty of NTUEE as an Associate Professor since 1991. His research interests include medical informatics, magnetic resonance imaging, and man-machine interface for disables.

Dr. Chen is a member of SMRM and AAPM. 